\title{
G.J.R. Cook, M.N. Maisey, K.E. Britton and V. Chengazi, (eds.): Clinical nuclear medicine, 4th edition.
}

\author{
Hodder Arnold, London, 2006. Hardcover, 896 pp. Price: $£$ 145.00, ISBN 0-340-812397
}

\section{P. M. Stokkel}

Published online: 10 July 2007

(C) Springer-Verlag 2007

Developments over recent years have forced workers in the field of nuclear medicine to update approaches, guidelines and clinical applications for PET and other aspects of molecular imaging regularly. In the fourth edition of Clinical Nuclear Medicine, the editors have done a great job, as the book presents topics of current importance that are relevant to the practising clinician with a problemoriented approach.

This book contains 21 chapters, 879 pages and a wellorganised index and is subdivided into three large sections. All chapters are written by highly esteemed colleagues, which is reflected in the excellent quality of the presentations and illustrations. Section A is dedicated to clinical topics, such as molecular imaging, principles of radionuclide therapy, imaging of infection and inflammation, paediatric imaging and sentinel node imaging. In addition to basic principles, each chapter nicely presents protocols and clinical studies. Furthermore, the tables are complete and provide a clear overview of imaging and treatmentrelated aspects, such as causes of false interpretations on PET scans, response criteria, biological applications of tracers, etc. Section B is dedicated to clinical systems, such as diseases of the head and neck, diseases of the cardiovascular system, diseases of the gastrointestinal tract and endocrine disorders. Most of the chapters are richly illustrated with black and white images or figures, while colour plates are provided for some topics. The position of these colour plates in the book is somewhat surprising, as

M. P. M. Stokkel ( $\bowtie)$

Leiden, The Netherlands

e-mail: M.P.M.Hagendoorn@lumc.nl the first group of plates is right in the middle of the chapter on cardiovascular diseases (between pp. 168 and 169) and the second group of plates, between salivary pathology and endocrine diseases (between pp. 487 and 488). It would have been more sophisticated to put these plates immediately before or after the index. Nevertheless, the quality of these illustrations is excellent. In the third section, some technical topics are discussed, as is nowadays inevitable in books dedicated to nuclear medicine. The first chapter in this part is related to pitfalls and artefacts in PET imaging, and the second addresses diagnostic accuracy and costeffectiveness; the author has provided an informative description ranging from basic statistical and epidemiological aspects to cost-benefit analysis. In chapter 20, the authors present a complete overview on radionuclides, with emphasis on interactions and reactions and the development of new radiopharmaceuticals for SPECT and PET. In the last chapter, technology and instrumentation are clearly described. Solid state and other detectors are summarised, and image registration and reconstruction techniques are presented and illustrated with good-quality figures.

The editors have stated that this book will be invaluable not only to practising and trainee nuclear medicine physicians and radiologists, but also to other groups of clinicians using nuclear medicine methods to investigate their patients, particularly cardiologists and oncologists. In my opinion, they have succeeded in their aim by combining easily readable chapters, high-quality figures and images and complete tables and reference lists. Therefore, this book is highly recommended, especially as a textbook for those who are involved in clinical nuclear medicine. Congratulations are due to the editors of and contributors to this work. 